\title{
Splenic Artery Embolization as a bridge to Splenectomy in severe Transfusion-Refractory Thrombocytopenia Secondary to Myelodysplastic Syndrome
}

\author{
Noa G. Holtzman ${ }^{1}$, Vu Duong ${ }^{1}$, Nader Hanna ${ }^{2}$, Howard M. Richard ${ }^{3}$, Ashkan Emadi ${ }^{*}$ \\ ${ }^{1}$ University of Maryland School of Medicine, Marlene \& Stewart Greenebaum Comprehensive Cancer Center, Baltimore, MD \\ 21201 \\ ${ }^{2}$ University of Maryland School of Medicine, Division of General and Oncologic Surgery \\ ${ }^{3}$ University of Maryland School of Medicine, Department of Diagnostic Imaging, Division of Interventional Radiology
}

*Corresponding author: Ashkan Emadi, M.D., Ph.D, Associate Professor of Medicine, Pharmacology and Experimental Therapeutics, Director, Hematology and Medical Oncology Fellowship Program, University of Maryland, School of Medicine, Marlene and Stewart Greenebaum Comprehensive Cancer Center, 22 South Greene Street, Room N9E24, Baltimore, Maryland 21201, USA, Tel: 410-328-2596, Fax: 410-328-6896; E-mail: aemadi@umm.edu

\begin{abstract}
Transfusion-refractory thrombocytopenia is an important clinical challenge in the treatment of patients with myelodysplastic syndrome. Hypersplenism can play a major role in splenic platelet sequestration and transfusion refractoriness, and though rare in myelodysplastic syndrome, it can commonly be seen in a wide range of other benign and malignant conditions. Splenectomy has been an effective approach in management of hypersplenism-induced cytopenias, however those who are unresponsive to platelet transfusions are not surgical candidates making this an unfeasible treatment option. There has been increasing interest in the therapeutic value of the less invasive procedure of splenic artery embolization. Here we report the first case of a patient with myelodysplastic syndrome complicated by hypersplenism and severe transfusion-refractory thrombocytopenia in which a splenic artery embolization was successfully used as a bridge to splenectomy, which resulted in platelet transfusion responsiveness and correction of her thrombocytopenia. Reversal of severe thrombocytopenia has multiple important therapeutic values including allowing patients to safely continue receiving myelosuppressive chemotherapy treatments which are sometimes delayed or dose-adjusted due to cytopenias, safely undergo surgical interventions and also open the door for candidacy for stem cell transplantation. In conclusion, splenic artery embolization with or without subsequent splenectomy is highly recommended as a safe therapeutic option in patients with hematologic malignancies.
\end{abstract}

Received Date: September 30, 2016

Accepted Date: October 28, 2016

Published Date: November 01, 2016

Citation: Emadi, A., et. al. Splenic Artery Embolization as a bridge to Splenectomy in severe Transfusion-Refractory Thrombocytopenia Secondary to Myelodysplastic Syndrome. (2016) Int J Hematol Ther 2(2): $1-5$.

DOI: $10.15436 / 2381-1404.16 .012$

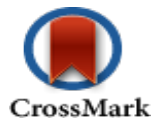

\section{Introduction}

Myelodysplastic syndrome (MDS) is a heterogeneous group of hematologic disorders that are defined by dysplastic, ineffective hematopoiesis ${ }^{[1]}$. It can manifest as cytopenia of a single blood cell lineage or of multiple. The goal of treatment for lower-risk MDS is usually palliative, focusing on symptom management and alleviation of transfusion burden. High-risk disease is associated with high chance of transformation to acute myeloid leukemia (AML) and short overall survival, thus young patients with few comorbidities and higher-risk disease are candidates for more aggressive curative treatment including allogeneic stem cell transplantation. In rare cases, MDS can be associated with hypersplenism ${ }^{[2]}$. Splenomegaly is more commonly seen in myeloproliferative neoplasms (MPN) such as primary myelofibrosis. When splenomegaly is seen with MDS, it is usually in cases

Copyrights: () 2016 Emadi, A. This is an Open access article distributed under the terms of Creative Commons Attribution 4.0 International License. 
in which the disease is associated with bone marrow fibrosis ${ }^{[3]}$. Hypersplenism can also be seen in a wide range of non-malignant conditions such as liver disease, hematologic disorders like thalassemia and hereditary spherocytosis, infectious diseases such as malaria and visceral leishmaniasis, as well as infiltrative diseases $^{[4,5]}$. Cytopenias secondary to hypersplenism have been reported in the literature to successfully resolve in response to interventions focused at shrinking or eliminating the spleen such as splenic radiation, splenic artery embolization or splenectomy ${ }^{[6,7]}$. An important clinical challenge arises when a severely thrombocytopenic patient who is transfusion-refractory requires an urgent surgical procedure. One possible treatment option is splenic artery embolization, which can serve as a safe alternative to splenectomy, and which has been shown to lead to a rapid increase in platelet count and transfusion responsiveness, opening the window for surgical intervention.

Here we report the first case in the literature to our knowledge of a patient with myelodysplastic syndrome complicated by hypersplenism and transfusion-refractory thrombocytopenia in which a splenic artery embolization was successfully used as a bridge to splenectomy.

\section{Case Report}

A 36-year-old woman with no past medical history presented to an outside facility with two weeks of fatigue, increased bruising, dyspnea, weakness and night sweats. She was found to have significant anemia and thrombocytopenia, and was transferred to our facility for further work up. On arrival, the patient was afebrile and hemodynamically stable. Her physical exam revealed scattered petechiae and palpable splenomegaly. Laboratory evaluation was notable for a white blood cell count of 6.1 $\mathrm{K} / \mathrm{mcL}$, hemoglobin of $8.2 \mathrm{~g} / \mathrm{dL}$, hematocrit of $23.8 \%$, platelet count of $4 \mathrm{~K} / \mathrm{mcL}$ and an elevated lactate dehydrogenase to 1042 units/L. After undergoing bone marrow biopsy, patient was diagnosed with high-grade myelodysplastic syndrome, refractory anemia with excess blasts-1 (RAEB-1) with moderate reticulin fibrosis of the bone marrow. Peripheral blood fluorescent in situ hybridization (FISH) analysis revealed presence of $\operatorname{del}(7 q)$ in $8 \%$ of cells. Mutational analysis was negative for JAK2-V617F, MPL and calreticulin mutations. The patient was initiated on treatment for her MDS with the DNA methyltransferase inhibitor azacitidine $75 \mathrm{mg} / \mathrm{m}^{2}$ intravenously daily for seven days every four weeks. Her hospitalization was complicated by development of severe transfusion-refractory thrombocytopenia, with platelet values consistently remaining below $10 \mathrm{~K} / \mathrm{mcL}$ despite daily human leukocyte antigen (HLA)-matched platelet transfusions. The patient had worsening splenomegaly, with her spleen size increasing from $18 \times 7 \times 16 \mathrm{~cm}^{3}$ to $20 \mathrm{~cm}$ in length at two weeks, and $28 \mathrm{~cm}$ in length at 8 weeks (Figure 1). The patient underwent an extensive infectious and rheumatologic work up for her splenomegaly, all of which was unremarkable.
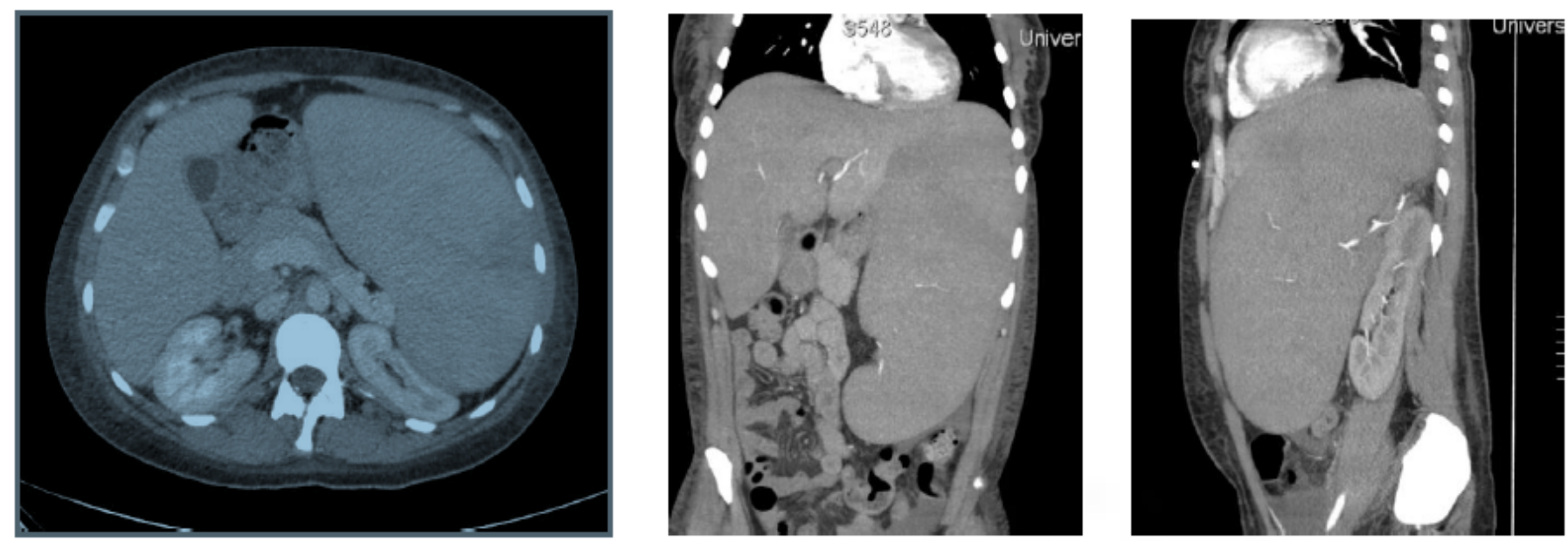

Figure 1: left: Computed tomography images of the abdomen, transverse view exhibiting significant splenomegaly, middle: Coronal view, right: Sagittal view.

Our patient's course was further complicated by menorrhagia and retinal vitreous hemorrhages secondary to her severe thrombocytopenia. Several pharmacologic approaches were attempted, including a trial of intravenous immunoglobulin (IVIG) with a fourteen-day course of prednisone $1 \mathrm{mg} / \mathrm{kg}$ /day and a twoweek trial of the thrombopoeitin receptor agonist eltrombopag $75 \mathrm{mg}$ daily. For her vaginal bleeding, the gynecology service was consulted and patient received a variety of hormonal treatments as well as the anti-fibrinolytic aminocaproic acid, resulting in mild improvement in her vaginal bleeding. Transvaginal ultrasound showed no specific abnormalities, except for a thickened endometrium consistent with secretory phase endometrium and hemorrhage. The surgical oncology team was consulted for evaluation for potential splenectomy, however given her continued refractoriness to platelet transfusions, she was deemed too high-risk for splenectomy. Therefore, splenic radiation treatment was pursued with the intention of causing splenic infarction and shrinkage of splenic volume. Our patient then underwent six sessions of biweekly splenic radiation treatments with a cumulative radiation dose of 4.5 Gray. Computed Tomography (CT) scan of the spleen post-radiation showed a large area of infarction measuring $15 \times 3.5 \mathrm{~cm}^{3}$, and a smaller area in the lower pole measuring $4 \mathrm{~cm}$ in length. After waiting several weeks to allow for the full potential effects of radiation, her platelet count unfortunately did not respond.

For her MDS, our patient was evaluated for hematopoietic stem cell transplantation. She was deemed not a candidate for transplantation at the time of evaluation due to her severe transfusion-refractory thrombocytopenia and her high risk of bleeding in the pre-engraftment period. When thought to have 
exhausted all resources, based on a few case reports found in the literature of splenic artery embolization in cases of other benign hematologic diseases and hypersplenism causing thrombocytopenia, our patient was referred for splenic artery embolization to be used as a pre-operative transient procedure to allow for platelet count response in preparation for splenectomy. The splenic artery embolization was completed using a 12-mm Amplatzer II plug, which successfully slowed the blood flow to the middle and distal parts of the splenic artery (Figures 2 and 3). The procedure was well tolerated without complications, and immediately post-embolization our patient's platelet count improved to $26 \mathrm{~K} / \mathrm{mcL}$. Patient received a platelet transfusion to which she was responsive for the first time with her platelets further increasing to $86 \mathrm{~K} / \mathrm{mcL}$, above the threshold for which she could undergo a splenectomy safely. A laparoscopic hand-assisted splenectomy was performed the following day without complication. Post-splenectomy, patient experienced post-operative pain which was well controlled by oral analgesics. Post-operative course was also complicated by development of atrial fibrillation which was self-limited and resolved with medical management. She received the appropriate post-splenectomy vaccinations including meningococcal, Haemophilus influenzae type B and pneumococcal vaccines. Our patient was discharged home for the first time after four months in the hospital and became a candidate for Bone Marrow Transplantation (BMT), for which further workup would be pursued as an outpatient. Unfortunately, our patient's disease transformed to AML later while she was undergoing evaluation for BMT. Her thrombocytopenia also recurred approximately six months post-splenectomy while undergoing treatment for AML, though she remained responsive to platelet transfusions.
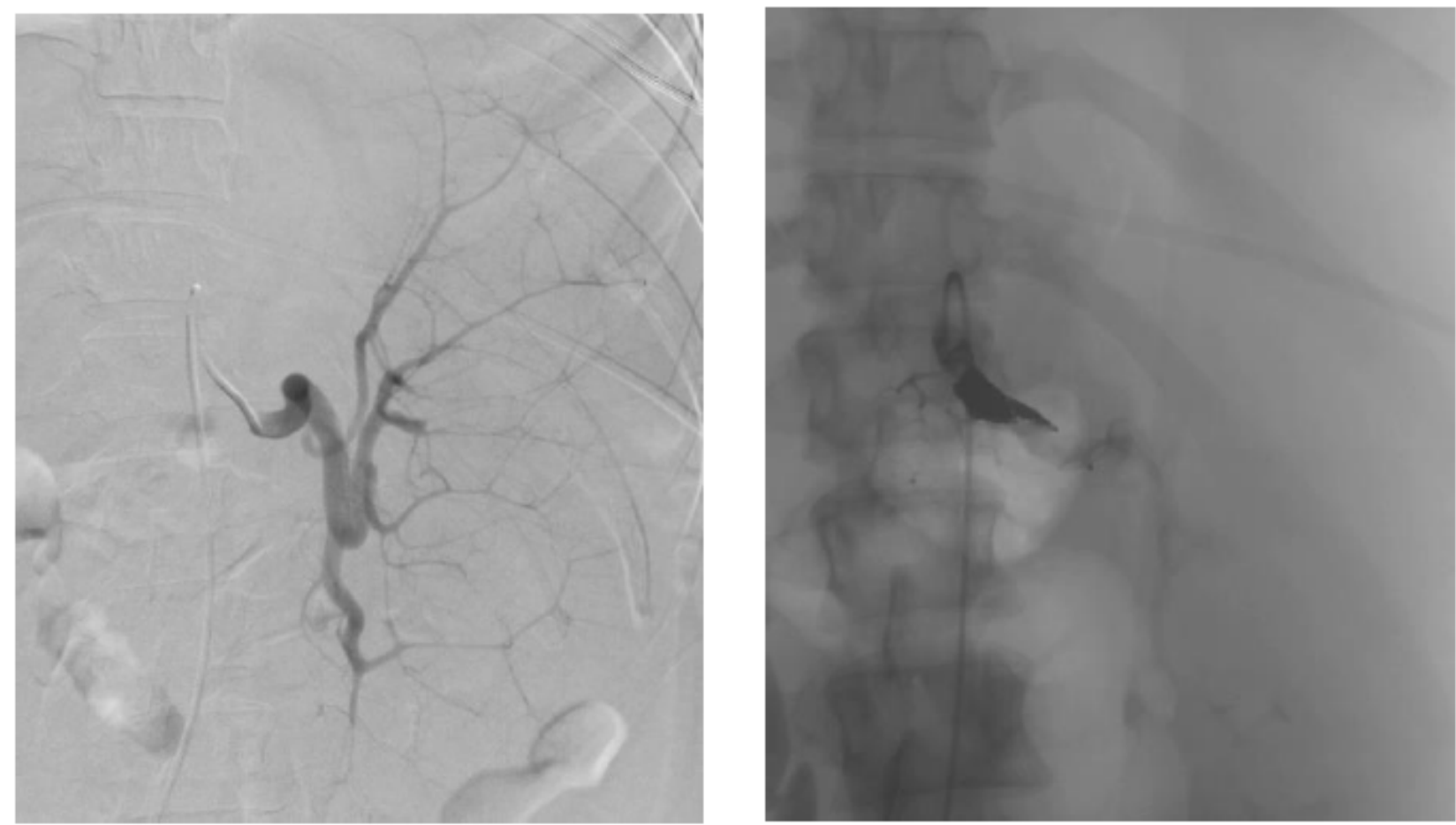

Figure 2: left: Pre-embolization splenic artery vasculature to a hypertrophied spleen.

Figure 3: right: Post-embolization, vasculature is seen completely blocked off by the Amplatzer plug inserted to the main splenic artery.

\section{Discussion}

Transfusion-refractory thrombocytopenia is an important clinical challenge that is commonly seen in MDS and other hematologic malignancies. In MDS, thrombocytopenia is seen in approximately $45-65 \%$ of cases $^{[8]}$, the severity of which increases with higher-risk disease ${ }^{[9]}$. Hemorrhagic complications are a major cause of morbidity and mortality in the MDS population, with estimated incidence of $14-24 \%$ of MDS-related deaths, only second to infection ${ }^{[10]}$. Management of this thrombocytopenia is complicated as there are several etiologies of thrombocytopenia in these patients that at times can overlap including (1) platelet HLA-alloimmunization; (2) chemotherapy-related bone marrow suppression; and (3) splenic sequestration of platelets. The short lifespan of platelets is also a limiting factor in its treatment, as it leads an increased requirement of frequent transfusions, which alone are associated with a significant risk of adverse effects as well as affecting the quality of life of the patient. Severe thrombocytopenia can also lead to delay or dose-reduction of chemotherapies or prevent candidacy for bone marrow transplantation. Treatment approaches in such cases aim at pharmacologically controlling thrombocytopenia-induced bleeding as well as causing shrinkage of the spleen to prevent continued platelet sequestration. Medical management includes use of medications that can help bleeding subside such as antifibrinolytics like aminocaproic acid ${ }^{[11]}$. There has been increased interest in the use of thrombopoeitin receptor agonists such as eltrombopag and romiplostim in myelodysplastic syndrome, which have shown efficacy in treatment of thrombocytopenia in chronic Idiopathic Thrombocytopenic Purpura (ITP) ${ }^{[12,13]}$. Though studies suggest successful increase in platelet counts and decreased bleeding, there is concern over increased risk for 
progression to acute myeloid leukemia, and more research is needed to assess for safe use of these agents in myelodysplastic syndrome and other malignant myeloid disorders ${ }^{[14,15]}$. If an immune component is suspected, IVIG and other immunosuppressive drugs such as steroids can be tried. Platelet alloimmunization-related transfusion refractoriness remains a significant challenge to treat, and has been shown to be refractory to treatment with IVIG and other immunosuppressants ${ }^{[15,17]}$. For hypersplenism-related thrombocytopenia, radiation is useful to induce splenic injury and infarction, but its effect can take weeks to manifest which is time that these patients usually do not have. In cases refractory to medical management and radiation, surgical intervention with splenectomy becomes standard of care and perhaps the best therapeutic option. These patients are, however, unfortunately not surgical candidates in these clinical scenarios due to their thrombocytopenia which can lead to life threatening bleeding if taken to the operating room.

Splenectomy has been shown to be an effective therapeutic option in the management of hypersplenism-induced cytopenia $^{[7]}$. Splenectomy is therapeutically pursued for this indication in a wide range of conditions including ITP, hereditary spherocytosis, and cirrhosis ${ }^{[7,18-20]}$. Many investigators have looked into less invasive alternatives such as splenic artery embolization. Splenic Artery Embolization (SAE) was first performed in 1973 and its therapeutic indications have since expanded. The success of splenic artery embolization in correcting cytopenias is due to the decreased rate of consumption of blood cells in the spleen from the blocked off blood flow to the spleen $^{[6]}$. Improvement in cytopenias are seen when the amount of infarcted spleen exceeds $50 \%{ }^{[4,21]}$, with the increase in platelet counts suggested to be correlated with the size of splenic infarction and splenic volume loss post embolization ${ }^{[6]}$. In one study of six patients who underwent splenic artery embolization due to hypersplenism, the peak platelet counts were achieved at one month post-embolization ${ }^{[6]}$. Size of splenic infarction was also associated with increased adverse effects such as splenic abscess development, duration of abdominal pain and risk of post embolization syndrome which is associated with fever, leukocytosis and abdominal pain ${ }^{[4,22]}$. The procedure's full therapeutic potential has been increasingly studied over just the past decade, with the majority of literature reported in liver disease with cirrhosis and portal hypertension-related hypersplenism. It has been reported as both a palliative and curative procedure in treatment of pathologic hyperslenism as seen in cases of chronic ITP ${ }^{[23,24]}$, paroxysmal nocturnal hemoglobinuria ${ }^{[25,26]}$, thalassemia ${ }^{[27,28]}$, gaucher's disease ${ }^{[29,30]}$, cirrhosis ${ }^{[21,31,32]}$, hereditary spherocytosis ${ }^{[33]}$, and renal transplant recipients ${ }^{[34]}$. SAE is even now being advocated as indicated in cases of cytopenias limiting treatment options in cancer patients such as colorectal, pancreatic or hepatocellular carcinoma, where treatment with marrow suppressive chemotherapeutics are vital ${ }^{[35]}$.

Many studies have explored the risks and benefits of SAE alone compared to splenectomy. Potential adverse effects from splenectomy include surgical complications and high morbidity due to infections with encapsulated bacteria ${ }^{[36,37]}$. Potential side effects of splenic artery embolization include post-embolization syndrome, splenic abscesses, septicemia, splenic rupture and pneumonia ${ }^{[38]}$. The advantages of embolization include its less invasive nature, and that the remaining healthy splenic tissue can provide immune protection ${ }^{[6]}$. One randomized trial in a group of cirrhotic patients, showed that both procedures were equally effective in cytopenia reversal and allowing for transfusion-responsiveness, however the rise in platelet count was greater in those who underwent splenectomy ${ }^{[39]}$. In our patient case, the benefits outweighed the risk of pursuing splenectomy in terms of both therapeutic and quality of life effects, as this definitive treatment corrected her transfusion-refractory thrombocytopenia, tremendously alleviated her significant abdominal pain and distension, and enhanced the chance for a potential future stem cell transplantation ${ }^{[40]}$. Additionally, this allowed for our patient to require less frequent laboratory count checks and transfusions than she required pre-intervention. Most importantly, her new platelet-transfusion responsiveness post-splenectomy allowed for eligibility for stem cell transplantation, which would be her only potential long-term curative treatment option.

\section{Conclusion}

To the best of our knowledge, this is the first reported case of splenic artery embolization used as a bridge to splenectomy in a patient with MDS. As transfusion-refractory thrombocytopenia is seen commonly in this patient population and treatment options are limited, splenic artery embolization with or without subsequent splenectomy is highly recommended as a safe therapeutic option. More research is encouraged to characterize the long term outcomes of embolization followed by splenectomy as a therapeutic solution for hypersplenism-related cytopenias in the hematologic malignancies.

Conflict of Interest: None of the authors report any relevant financial conflict of interest. 


\section{References}

1. Hofmann, W.K., Koeffler, H.P. Myelodysplastic syndrome. (2005) Annu Rev Med 56: 1-16.

2. Kraus, M.D., Bartlett, N.L., Fleming, M.D., et al. Splenic pathology in myelodysplasia: a report of 13 cases with clinical correlation. (1998) Am J Surg Pathol 22(10): 1255-1266.

3. Verhoef, G.E., De Wolf-Peeters, C., Ferrant, A., et al. Myelodysplastic syndromes with bone marrow fibrosis: a myelodysplastic disorder with proliferative features. (1991) Ann Hematol 63(5): 235-241.

4. Boyer, T.D., Habib, S. Big spleens and hypersplenism: fix it or forget it?. (2015) Liver Int 35(5): 1492-1498.

5. Gallina, V., Binazzi, R., Golemi, A., et al. Imported visceral leishmaniasis - unexpected bone marrow diagnosis in a patient with fever, pancytopenia, and splenomegaly. (2014) Am J Blood Res 4(2): 101105.

6. Pisco, J.M., Reis, A.P., Fernandes, M.O., et al. Percutaneous splenic embolization of the splenic artery in the treatment of hypersplenism. (1992) Acta Med Port 5(8): 433-436.

7. Watanabe, Y., Horiuchi, A., Yoshida, M., et al. Significance of laparoscopic splenectomy in patients with hypersplenism. (2007) World J Surg 31: 549-555.

8. Kantarjian, H., Giles, F., List, A., et al. The incidence and impact of thrombocytopenia in myelodysplastic syndromes. (2007) Cancer 109(9): 1705-1714.

9. Bryan, J., Jabbour, E., Prescott, H., et al. Thrombocytopenia in patients with myelodysplastic syndromes. (2010) Semin Hematol 47(3): 274-280.

10. Gupta, P., LeRoy, S.C., Luikart, S.D., et al. Long-term blood product transfusion support for patients with myelodysplastic syndromes (MDS): cost analysis and complications. (1999) Leuk Res 23(10): 953959.

11. Kalmadi, S., Tiu, R., Lowe, C., et al. Epsilon aminocaproic acid reduces transfusion requirements in patients with thrombocytopenic hemorrhage. (2006) Cancer 107(1): 136.

12. Bussel, J.B., Cheng, G., Saleh, M.N., et al. Eltrombopag for the Treatment of Chronic Idiopathic Thrombocytopenic purpura. (2007) N Engl J Med 357: 2237-2247.

13. Kuter, D.J., Rummel, M., Boccia, R., et al. Romiplostim or standard of care in patients with immune thrombocytopenia. (2010) N Engl J Med 363: 1889-1899.

14. Kantarjian, H., Fenaux, P., Sekeres, M.A., et al. Safety and efficacy of romiplostim in patients with lower-risk myelodysplastic syndrome and thrombocytopenia. (2010) J Clin Oncol 28(3): 437-444.

15. Platzbecker, U., Sekeres, M.A., Kantarjian, H., et al. Relationship of different platelet response criteria and patient outcomes in a romiplostim myelodysplastic syndromes trial. (2014) Leukemia 28(12): 2418 2421.

16. Kickler, T., Braine, H.G., Piantadosi, S., et al. A randomized, placebo-controlled trial of intravenous gammaglobulin in alloimmunized thrombocytopenic patients. (1990) Blood 75(1): 313-316.

17. Schiffer, C.A., Hogge, D.E., Aisner, J., et al. High-dose intravenous gammaglobulin in alloimmunized platelet transfusion recipients. (1984) Blood 64(4): 937-940.

18. Al-Salem, A.H., Nasserulla, Z. Splenectomy for children with thalassemia. (2002) Int Surg 87(4): 269-273.

19. Bo, W., He-Shui, W., Guo-Bin, W., et al. Laparoscopy splenectomy for massive splenomegaly. (2013) J Invest Surg 26(3): 154-157.

20. Kumar, S., Diehn, F.E., Gertz, M.A., et al. Splenectomy for immune thrombocytopenic purpura: long-term results and treatment of postsplenectomy relapses. (2002) Ann Hematol 81(6): 312-319.
21. Omer, S., Zara, O., Iacobescu, C., et al. Partial splenic embolization for hypersplenism in cirrhotic patients. A case series. (2014) J Gastrointestin Liver Dis 23(2): 215-218.

22. Guan, Y.S., Hu, Y. Clinical application of partial splenic embolization. Scientific World Journal 2014: 961345.

23. Kimura, F., Itoh, H., Ambiru, S., et al. Long-term results of initial and repeated partial splenic embolization for the treatment of chronic idiopathic thrombocytopenic purpura. (2002) AJR Am J Roentgenol 179(5): 1323-1326.

24. Miyazaki, M., Itoh, H., Kaiho, T., et al. Partial splenic embolization for the treatment of chronic idiopathic thrombocytopenic purpura. (1994) AJR Am J Roentgenol 163(1): 123-126.

25. Araten, D.J., Iori, A.P., Brown, K., et al. Selective splenic artery embolization for the treatment of thrombocytopenia and hypersplenism in paroxysmal nocturnal hemoglobinuria. (2014) J Hematol Oncol 7: 27.

26. Krishnan, S.K., Hill, A., Hillmen, P., et al. Improving cytopenia with splenic artery embolization in a patient with paroxysmal nocturnal hemoglobinuria on eculizumab. (2013) Int J Hematol 98(6): 716-718.

27. Pinca, A., Di Palma, A., Soriani, S., et al. Effectiveness of partial splenic embolization as treatment for hypersplenism in thalassaemia major: a 7-year follow up. (1992) Eur J Haematol 49(2): 49-52.

28. Politis, C., Spigos, D.G., Georgiopoulou, P., et al. Partial splenic embolization for hypersplenism of thalassaemia major. (1987) $\mathrm{Br} \mathrm{Med}$ J 294(6573): 665-667.

29. Pena, A.H., Kaplan, P., Ganesh, J., et al. Partial splenic embolization in a child with Gaucher disease, massive splenomegaly and severe thrombocytopenia. (2009) Pediatr Radiol 39(9): 1006-1009.

30. Thanopoulos, B.D., Frimas, C.A. Partial splenic embolization in the management of hypersplenism secondary to Gaucher disease. (1982) J Pediatr 101(5): 740-743.

31. Hidaka, H., Kokubu, S., Saigenji, K., et al. Restoration of thrombopoietin production after partial splenic embolization leads to resolution of thrombocytopenia in liver cirrhosis. (2002) Hepatol Res 23(4): $265-$ 273

32. Zhu, K., Meng, X., Qian, J., et al. Partial splenic embolization for hypersplenism in cirrhosis: a long-term outcome in 62 patients. (2009) Dig Liver Dis 41(6): 411-416.

33. Kimura, F., Ito, H., Shimizu, H., et al. Partial splenic embolization for the treatment of hereditary spherocytosis. (2003) AJR Am J Roentgenol 181(4): 1021-1024.

34. Gerlock, A.J.Jr., MacDonell, R.C.Jr., Muhletaler, C.A., et al. Partial splenic embolization for hypersplenism in renal transplantation. (1982) AJR Am J Roentgenol 138(3): 451-456.

35. Kauffman, C.R., Mahvash, A., Kopetz, S., et al. Partial splenic embolization for cancer patients with thrombocytopenia requiring systemic chemotherapy. (2008) Cancer 112(10): 2283-2288.

36. Morgan, T.L., Tomich, E.B. Overwhelming post-splenectomy infection (OPSI): a case report and review of the literature. (2012) J Emerg Med 43(4): 758-763.

37. Sakai, T., Shiraki, K., Inoue, H., et al. Complications of partial splenic embolization in cirrhotic patients. (2002) Dig Dis Sci 47: 388391.

38. Spigos, D.G., Jonasson, O., Mozes, M., et al. Partial splenic embolization in the treatment of hypersplenism. (1979) AJR Am J Roentgenol 132: 777-782.

39. Amin, M.A., el-Gendy, M.M., Dawoud, I.E., et al. Partial splenic embolization versus splenectomy for the management of hypersplenism in cirrhotic patients. (2009) World J Surg 33(8): 1702-1710.

40. Akpek, G., Pasquini, M.C., Logan, B., et al. Effects of splenectomy, splenic irradiation, and splenomegaly on early outcomes after hematopoietic cell transplantation. (2013) Bone Marrow Transplant 48(6): 825-831.
Ommega Online Publisher

International Journal of Hematology \& Therapy

Short Title : Int J Hematol Ther

\section{ISSN: 2381-1404}

E-mail : hematology.therapy@ommegaonline.org

website: www.ommegaonline.org 